\title{
Effect of percutaneous transluminal coronary angioplasty on arrhythmias complicating angina
}

\author{
ADENIYI O MOLAJO, GREGORY D SUMMERS, * DAVID H BENNETT \\ From the Regional Cardiac Centre, Wythenshawe Hospital, Manchester
}

SUMMARY Four patients who had stenosis of a single major coronary artery which was treated by percutaneous transluminal coronary angioplasty are described. Three had exercise induced myocardial ischaemia complicated by ventricular tachycardia, fibrillation, and sinus bradycardia, respectively. Asystole developed in a fourth patient who had spontaneous chest pain. After successful percutaneous transluminal coronary angioplasty these arrhythmias did not recur spontaneously or on treadmill exercise testing. Percutaneous coronary angioplasty can be effective in preventing arrhythmias complicating acute myocardial ischaemia secondary to stenosis of a single major coronary artery.

Rhythm disturbance is a not uncommon complication of coronary artery disease. New data derived from angiographic and haemodynamic studies of patients resuscitated after sudden cardiac death had been imminent showed that in most $(94 \%)$ the diameter of at least one major coronary artery had been reduced by $70 \%$ or more. ${ }^{1}$ Antiarrhythmic drugs are usually given to prevent recurrence of arrhythmia. Alternative treatments are aneurysmectomy in selected cases, ${ }^{23}$ coronary artery bypass grafting, ${ }^{4}$ and endocardial excision guided by ventricular mapping in some others. ${ }^{5}$

We describe our experience with four patients who had exertional angina complicated by arrhythmia. They were all successfully managed by percutaneous transluminal coronary angioplasty.

\section{Case reports}

CASE 1

A 34 year old man with a three month history of exertional angina was referred to a district general hospital. His resting electrocardiogram was normal. $\mathrm{He}$ was receiving atenolol, isosorbide mononitrate, and nifedipine. A Master's two-step exercise test ${ }^{6}$

Requests for reprints to Dr A O Molajo, Regional Cardiac Centre, Wythenshawe Hospital, Southmoor Road, Wythenshawe, Manchester M239LT.

^Present address: Northampton General Hospital.

Accepted for publication 22 July 1985 was performed without electrocardiographic monitoring. After five minutes of exercise ventricular fibrillation developed. $\mathrm{He}$ was successfully defibrillated. Serial electrocardiograms and serum concentrations of cardiac enzymes were normal. $\mathrm{He}$ was subsequently transferred to our hospital. Left heart catheterisation showed normal left ventricular function and severe proximal stenosis of the left anterior descending coronary artery. Successful coronary angioplasty was performed five days later.

He was maintained on nifedipine, enteric coated aspirin, and dipyridamole. Repeat maximal symptom limited treadmill exercise testing four days after the angioplasty which followed the standard Bruce protocol $^{7}$ was terminated at ten minutes because of fatigue. The heart rate was then 170 beats per minute and systemic systolic pressure was $146 \mathrm{~mm} \mathrm{Hg}$. There was ST depression of $0.7 \mathrm{~mm}$ in lead V5. No arrhythmia was seen. He has been free of angina and arrhythmia for more than twelve months.

\section{CASE 2}

A 34 year old man with a two month history of exertional angina was admitted to a district general hospital after an episode of severe chest pain complicated by syncope. He was receiving atenolol, verapamil, and nitrates. Serial electrocardiograms and measurement of serum concentrations of cardiac enzymes did not show changes typical of myocardial infarction. Treadmill exercise testing was performed by the standard Bruce protocol. After 4 minutes 18 seconds of exercise testing ventricular tachycardia 
developed. Before the onset of ventricular tachycardia he had ST depression of $2.2 \mathrm{~mm}$ in lead aVF. He was successfully defibrillated and transferred to our hospital.

Left heart catheterisation showed normal left ventricular function and a severe proximal stenosis of the left anterior descending coronary artery. Coronary angioplasty was successfully performed. He was maintained on enteric coated aspirin and dipyridamole. Repeat treadmill exercise testing three days later by the standard Bruce protocol was terminated at 10 minutes 30 seconds because of fatigue. His heart rate was then 178 beats per minute, systemic systolic pressure was $210 \mathrm{~mm} \mathrm{Hg}$, and there was ST depression of $0.5 \mathrm{~mm}$ in lead V5 without evidence of ventricular irritability. He has remained free of angina and syncope for more than 12 months without antianginal or antiarrhythmic treatment.

\section{CASE 3}

A 49 year old woman had a long history of polymyositis. Previous peripheral nerve conduction studies had shown no abnormalities. She had a two year history of angina of effort and several years' history of hypertension. She was admitted to the casualty department with severe chest pain and became asystolic. She was successfully resuscitated with intravenous calcium, adrenaline, and temporary transvenous ventricular pacing. Serial measurements of serum concentrations of cardiac enzymes showed no evidence of myocardial infarction. Over the next few days she was dependent on a pacemaker.

Later persistent junctional rhythm with sinus arrest developed. Cardiac catheterisation showed good left ventricular function and a proximal right coronary artery stenosis at the origin of the sinus node artery. Right coronary angioplasty was successfully performed. Sinus rhythm returned 24 hours later. Repeat coronary angiography four months later showed minor irregularity of right coronary artery and she has remained in sinus rhythm without any further syncope.

\section{CASE 4}

A 60 year old man gave a history of classic exertional angina. An exercise test conducted according to the standard Bruce protocol was performed at another hospital. At the seventh minute of exercise, heart rate fell suddenly from 170 to 90 beats per minute. At this point he had ST depression of $1.2 \mathrm{~mm}$ in lead aVF. He was on oxprenolol, nifedipine, and isosorbide mononitrate. He was referred to this hospital for coronary angiography. This showed good left ventricular function and severe right coronary artery stenosis. Right coronary angioplasty was success- fully performed. He was maintained on enteric coated aspirin and dipyridamole. During repeat exercise testing three days later he was stopped by fatigue at 8 minutes 21 seconds at a heart rate of 174 beats per minute. There was no angina. The greatest degree of ST depression $(0.4 \mathrm{~mm})$ was seen in lead aVF. In contrast to the exercise test before angioplasty, there was no abrupt fall in heart rate during exercise. He has been free of symptoms for more than 16 months.

\section{Discussion}

In these four cases a disturbance in heart rhythm seemed to be associated with, and was presumably caused by, acute myocardial ischaemia-since arrhythmia occurred during episodes of chest pain and during exercise. After revascularisation had been achieved by transluminal coronary angioplasty the arrhythmia did not recur.

It seems reasonable to expect that prevention of ischaemia by coronary revascularisation would make a patient less susceptible to arrhythmia caused by acute ischaemia. There are a few reports of the efficacy of aortocoronary bypass grafting in abolishing ventricular arrhythmias precipitated by acute ischaemia ${ }^{48}$ Aortocoronary bypass grafting with or without aneurysmectomy, however, is seldom useful in the management of ventricular arrhythmias not caused by acute ischaemia. ${ }^{910}$ Whereas chronic ventricular arrhythmias in ischaemic heart disease are not necessarily abolished by aortocoronary bypass grafting, when ventricular arrhythmia is precipitated by acute ischaemia, revascularisation may be beneficial. Coronary angioplasty does not have to be followed by a period of very limited patient activity and therefore the tendency to arrhythmia after revascularisation may be assessed soon after angioplasty. Successful coronary angioplasty is unlikely to prevent arrhythmia not associated with acute ischaemia and antiarrhythmic drugs, ventriculotomy, aneurysmectomy, or pacing to prevent tachycardia may be required.

In case $\mathbf{4}$ there was an abrupt fall in heart rate during exercise. This is a recognised sign of ischaemia ${ }^{11}$ and was not found when the patient was exercised after coronary angioplasty. It should be noted that the patient was on a beta blocking drug in the exercise test before angioplasty but these drugs attenuate the chronotropic response to exercise rather than cause an abrupt fall in heart rate.

In case 3 there was a very severe right coronary artery stenosis proximal to the origin of the sinus node branch. There were no signs of sinus node dysfunction after coronary angioplasty. Coronary artery disease only accounts for a small proportion 
(perhaps $10-20 \%)^{11}$ of cases of sick sinus syndrome and the remainder are associated with degeneration, fibrosis, or amyloid infiltration of the sinus node. The return of sinus rhythm after coronary angioplasty in case 3, however, does suggest that ischaemia of the sinus node was the cause of sinus arrest in this case.

There are a few reports of coronary artery bypass grafting being an effective treatment for arrhythmias caused by acute ischaemia, but to our knowledge the successful use of coronary angioplasty for this purpose has not been reported before.

\section{References}

1 Weaver DW, Lorch GS, Alvarez HA, Cobb LA. Angiographic findings and prognostic indicators in patients resuscitated from sudden cardiac death. Circulation 1976; 54: 895-900.

2 Maloy WC, Arrant JE, Sowell BF, Hendrix GH. Left ventricular aneurysm of uncertain etiology with recurrent ventricular arrhythmias. $N$ Engl $\mathcal{F}$ Med 1971; 285: 662-3.

3 Thind GS, Blakemore WS, Zinsser HF. Ventricular aneurysmectomy for the treatment of recurrent ventric- ular tachyarrhythmia. Am $\mathcal{F}$ Cardiol 1971; 27: 690-4.

4 Bryson AL, Parisi A, Schechter E, Wolfson S. Lifethreatening ventricular arrhythmias induced by exercise. Cessation after coronary bypass surgery. $\mathrm{Am} \mathcal{f}$ Cardiol 1973; 32: 995-9.

5 Harken AH, Horowitz LN, Josephson ME. The surgical treatment of ventricular tachycardia. Ann Thorac Surg 1980; 30: 499-508.

6 Master AN, Rosenfeld I. Criteria for the clinical application of the "two-step" exercise test. $\mathcal{F} A M A$ 1961; 178: 283-9.

7 Bruce RA, Hornsten TR. Exercise stress testing in evaluation of patients with ischemic heart disease. Prog Cardiovasc Dis 1969; 11: 371-90.

8 Tommaso C, Kehoe R, Zheutlin T. Survivors of ischemic mediated sudden death. Clinical, angiographic and electrophysiologic features and response to therapy [Abstract]. Circulation 1982; 66 (suppl II): 25.

9 Mundth ED, Buckley MJ, De Sanctis RW, Daggett WM, Austen WG. Surgical treatment of ventricular irritability. I Thorac Cardiovasc Surg 1973; 66: 943-51.

10 Sami M, Chaitman BR, Bourassa MG, Charpin D, Chabot M. Long-term follow-up of aneurysmectomy for recurrent ventricular tachycardia or fibrillation. $\mathrm{Am}$ Heart $\mathcal{F}$ 1978; 96: 303-8.

11 Shaw DB. Sino-atrial dysfunction-sick sinus syndrome. In: Sleight P, Jones JV, eds. Scientific foundations of cardiology. London: Heinemann, 1983: 363-4. 